\title{
ON THE BASIS NUMBER OF THE COMPOSITION OF DIFFERENT LADDERS WITH SOME GRAPHS
}

\author{
MAREF Y. ALZOUBI AND M. M. M. JARADAT
}

Received 31 October 2004 and in revised form 9 June 2005

The basis number $b(G)$ of a graph $G$ is defined to be the least integer $k$ such that $G$ has a $k$-fold basis for its cycle space. In this paper, we investigate the basis number of the composition of paths and cycles with ladders, circular ladders, and Möbius ladders.

\section{Introduction}

The graphs considered in this paper are finite, undirected, simple, and connected. Most of the notations that follow can be found in [6] or [8]. Let $G=(V, E)$ be a graph, where $V$ and $E$ are the vertex and the edge sets of $G$, respectively. If $e_{1}, e_{2}, \ldots, e_{q}$ is an ordering of the edges in $G$, then any subset $S$ of edges corresponds to a $(0,1)$-vector $\left(a_{1}, a_{2}, \ldots, a_{q}\right)$ in the usual way, with $a_{i}=1\left(a_{i}=0\right)$ if and only if $e_{i} \in S\left(e_{i} \notin S\right)$. These vectors form a $q$ dimensional vector space $\left(Z_{2}\right)^{q}$ over the field $Z_{2}$. The vectors in $\left(Z_{2}\right)^{q}$ which correspond to the cycles in $G$ generate a subspace called the cycle space of $G$ denoted by $\mathscr{C}(G)$. We will say that the cycles themselves, rather than the vectors corresponding to them, generate $\mathscr{C}(G)$. It is known that for a connected graph $G$,

$$
\operatorname{dim} \mathscr{C}(G)=|E(G)|-|V(G)|+1 .
$$

The first important use of the basis number dates back to MacLane [11] when he made the connection between the basis number of a graph and the planarity. Thereafter, in 1981, Schmeichel [12] formalized the definition of the basis number of a graph as follows: a basis of a cycle space $\mathscr{C}(G)$ is called a $k$-fold basis if each edge of $G$ occurs in at most $k$ of the cycles in the basis. The basis number of $G$, denoted by $b(G)$, is the smallest integer $k$ such that $\mathscr{C}(G)$ has a $k$-fold basis.

In light of MacLane's ideas and Schmeichel's formal definition, we notice that studying the basis number of nonplanar graphs is more interesting than the planar ones.

Schmeichel investigated the basis number of certain important classes of nonplanar graphs, specifically, complete graphs and complete bipartite graphs. Then Banks and Schmeichel [5] proved that for $n \geq 7$, the basis number of $Q_{n}$ is 4 , where $Q_{n}$ is the $n$ cube. After that, many researchers were attracted to work on finding the basis number of 
special kinds of graphs, mainly, those that are obtained from different kinds of products of given graphs. We refer the interested readers to $[1,2,3,4,7,9,10]$.

The following results due to MacLane [11] and Hailat and Alzoubi [7] will be used frequently in our work.

Theorem 1.1 (MacLane). A graph $G$ is planar if and only if $b(G) \leq 2$.

Lemma 1.2 (Hailat and Alzoubi). Let $G$ be a graph with $p$ vertices and qedges. If $|C|$ denotes the length of the cycle $C$, and $\mathscr{B}=\left\{C_{1}, C_{2}, \ldots, C_{d}:\left|C_{i}\right| \geq r\right\}$ is a $k$-fold basis of $\mathscr{C}(G)$, then $r d \leq \sum_{i=1}^{d}\left|C_{i}\right| \leq k q$, where $d=\operatorname{dim} \mathscr{b}(G)$.

Definition 1.3. The composition (lexicographic) of two graphs $G_{1}\left(V_{1}, E_{1}\right)$ and $G_{2}\left(V_{2}, E_{2}\right)$, denoted by $G_{1}\left[G_{2}\right]$, is a graph with vertex set $V_{1} \times V_{2}$ and edge set $E\left(G_{1}\left[G_{2}\right]\right)=\left\{\left(u_{1}, v_{1}\right)\right.$ $\left(u_{2}, v_{2}\right): u_{1} u_{2} \in E_{1}$ or $u_{1}=u_{2}$ and $\left.v_{1} v_{2} \in E_{2}\right\}$.

It is worth mentioning that, in general, $G_{1}\left[G_{2}\right]$ and $G_{2}\left[G_{1}\right]$ are not isomorphic graphs since $\left|E\left(G_{1}\left[G_{2}\right]\right)\right|=p_{1} q_{2}+p_{2}^{2} q_{1}$ and $\left|E\left(G_{2}\left[G_{1}\right]\right)\right|=p_{2} q_{1}+p_{1}^{2} q_{2}$.

Definition 1.4. The ladder graph $L_{m}$ is a graph with vertex set $V\left(L_{m}\right)=\left\{u_{i}, v_{i}: 1 \leq i \leq m\right\}$ and edge set $E\left(L_{m}\right)=\left\{u_{i} u_{i+1}, v_{i} v_{i+1}: 1 \leq i \leq m-1\right\} \cup\left\{u_{i} v_{i}: 1 \leq i \leq m\right\}$. The circular ladder $\mathrm{CL}_{m}$ is a graph obtained from the ladder graph $L_{m}$ by adding the two edges $u_{m} u_{1}$, $v_{m} v_{1}$. The Möbius ladder $\mathrm{ML}_{m}$ is a graph obtained from the circular ladder $\mathrm{CL}_{m}$ by deleting two parallel curved edges and replace them by two edges that cross.

The main focus of this paper is to investigate the basis number of the composition of paths and cycles with ladders, circular ladders, and Möbius ladders.

\section{Main results}

In this section, we investigate the basis number of the compositions $P_{n}\left[L_{m}\right], P_{n}\left[\mathrm{CL}_{m}\right]$, $P_{n}\left[\mathrm{ML}_{m}\right], C_{n}\left[L_{m}\right], C_{n}\left[\mathrm{CL}_{m}\right]$, and $C_{n}\left[\mathrm{ML}_{m}\right]$, where $P_{n}$ is a path on $n$ vertices, $C_{n}$ is a cycle on $n$ vertices, $L_{m}$ denotes a ladder graph with $2 m$ vertices and $3 m-2$ edges, $\mathrm{CL}_{m}$ denotes a circular ladder graph with $2 m$ vertices and $3 m$ edges, and $\mathrm{ML}_{m}$ denotes a Möbius ladder graph with $2 m$ vertices and $3 m$ edges.

In this paper, we consider $P_{n}=123 \cdots n, C_{n}=123 \cdots n 1$, and the circular ladder $\mathrm{CL}_{m}$ as a graph obtained by drawing the two concentric $m$-cycles $u_{1} u_{2} \cdots u_{m} u_{1}$ and $v_{1} v_{2} \cdots v_{m} v_{1}$ in addition to the set of edges $\left\{u_{i} v_{i}: 1 \leq i \leq m\right\}$. Also, we consider the graph of the ladder $L_{m}$ as a graph obtained from the circular ladder $\mathrm{CL}_{m}$ by deleting the edges $u_{m} u_{1}$ and $v_{m} v_{1}$, and the graph of the Möbius ladder $\mathrm{ML}_{m}$ as a graph obtained from the circular ladder $\mathrm{CL}_{m}$ by deleting two parallel curved edges and replacing them by two edges that cross. For the sake of simplicity in our proofs, we consider $\mathrm{ML}_{m}$ to be obtained from $\mathrm{CL}_{m}$ by deleting the edges $u_{m} u_{1}$ and $v_{m} v_{1}$ and replacing them by the edges $u_{m} v_{1}$ and $v_{m} u_{1}$.

The graph of $P_{n}\left[L_{m}\right]$ has $2 m n$ vertices that occur in the following vertex set:

$$
V\left(P_{n}\left[L_{m}\right]\right)=\left\{\left(i, u_{j}\right),\left(i, v_{j}\right): 1 \leq i \leq n, 1 \leq j \leq m\right\} .
$$

It is well known that $\left|E\left(P_{n}\left[L_{m}\right]\right)\right|=\left|E\left(P_{n}\right)\right|\left|V\left(L_{m}\right)\right|^{2}+\left|V\left(P_{n}\right)\right|\left|E\left(L_{m}\right)\right|=4 m^{2}(n-1)$ $+3 m n-2 n$, and $\operatorname{dim} \mathscr{C}\left(P_{n}\left[L_{m}\right]\right)=\left|E\left(P_{n}\left[L_{m}\right]\right)\right|-\left|V\left(P_{n}\left[L_{m}\right]\right)\right|+1=4 m^{2}(n-1)+m n-$ $2 n+1$. 
The following identification of vertices plays an important role in our proofs:

$$
u_{m+1}=v_{m}, u_{m+2}=v_{m-1}, \ldots, u_{2 m-1}=v_{2}, u_{2 m}=v_{1}
$$

Thus, we may consider $V\left(P_{n}\left[L_{m}\right]\right)=\left\{\left(i, u_{j}\right): 1 \leq i \leq n, 1 \leq j \leq 2 m\right\}$. Following this notation, we look at the graph of $P_{n}\left[L_{m}\right]$ as a graph that consists of $n-1$ copies of the complete regular bipartite graph $K_{2 m, 2 m}$ in addition to the following sets of edges:

$$
\begin{gathered}
E_{1}=\left\{\left(i, u_{j}\right)\left(i, u_{j+1}\right): 1 \leq i \leq n, 1 \leq j \leq 2 m-1\right\}, \\
E_{2}=\left\{\left(i, u_{j}\right)\left(i, u_{2 m+1-j}\right): 1 \leq i \leq n, 1 \leq j \leq m-1\right\} .
\end{gathered}
$$

Note that each copy of $K_{2 m, 2 m}$ connects the vertex set $\left\{\left(i, u_{j}\right): 1 \leq j \leq 2 m\right\}$ with the vertex set $\left\{\left(i+1, u_{j}\right): 1 \leq j \leq 2 m\right\}$ for each $i$, where $1 \leq i \leq n-1$.

Theorem 2.1. For each $n \geq 2$ and $m \geq 5,3 \leq b\left(P_{n}\left[L_{m}\right]\right) \leq 4$. Moreover, $b\left(P_{n}\left[L_{m}\right]\right)=4$ for all $n \geq 2$ and $m \geq 7$.

Proof. It is clear that the graph of $P_{n}\left[L_{m}\right]$ is nonplanar, then by MacLane's theorem, we have $b\left(P_{n}\left[L_{m}\right]\right) \geq 3$. To prove that $b\left(P_{n}\left[L_{m}\right]\right) \leq 4$, we have to find a 4 -fold basis for the cycle space of $P_{n}\left[L_{m}\right]$. We define $\mathscr{B}_{(}\left(P_{n}\left[L_{m}\right]\right)=\mathscr{B}_{s} \cup \mathscr{B}_{1} \cup \mathscr{B}_{2}$, where $\mathscr{B}_{s}=\bigcup_{i=1}^{n-1} \mathscr{B}_{s i}$, $\mathscr{B}_{1}=$ $\bigcup_{i=1}^{n-1} \mathscr{B}_{1 i}$, and $\mathscr{B}_{2}=\cup_{i=1}^{n} \mathscr{B}_{2 i}$. The sets of cycles $\mathscr{B}_{s i}, \mathscr{B}_{1 i}$, and $\mathscr{B}_{2 i}$ are defined as follows:

$$
\begin{aligned}
& \mathscr{B}_{s i}=\left\{\left(i, u_{j}\right)\left(i+1, u_{k}\right)\left(i, u_{j+1}\right)\left(i+1, u_{k+1}\right)\left(i, u_{j}\right): 1 \leq j, k \leq 2 m-1\right\}, \\
& \mathscr{B}_{1 i}=\left\{\left(i, u_{1}\right)\left(i+1, u_{j}\right)\left(i+1, u_{j+1}\right)\left(i, u_{1}\right),\left(i+1, u_{2 m}\right)\left(i, u_{j}\right)\left(i, u_{j+1}\right)\left(i+1, u_{2 m}\right): 1 \leq\right. \\
& j \leq 2 m-1\}, \\
& \mathscr{B}_{2 i}=\left\{\left(i, u_{j}\right)\left(i, u_{2 m-j+1}\right)\left(i, u_{2 m-j}\right)\left(i, u_{j+1}\right)\left(i, u_{j}\right): 1 \leq j \leq m-1\right\} .
\end{aligned}
$$

For each $i, \mathscr{B}_{s i}$ is the Schmeichel basis for the $i$ th copy of $K_{2 m, 2 m}$ which is proved in Schmeichel [12, Theorem 4]. Then each $\mathscr{B}_{s i}$ is linearly independent, and since all the copies of $K_{2 m, 2 m}$ are edge-disjoint, we conclude that $\mathscr{H}_{s}$ is linearly independent.

For each $1 \leq i \leq n-1, \mathscr{B}_{1 i}$ is a basis for the cycle subspace of $\mathscr{C}\left(P_{n}\left[L_{m}\right]\right)$ corresponding to the planar subgraph of $P_{n}\left[L_{m}\right]$ obtained by pasting all the cycles of $\mathscr{S}_{1 i}$, which are 3 -cycles, at the common edges of the successive cycles. Thus the cycles of $\mathscr{S}_{1 i}$ are linearly independent for each $i$. Moreover, every 3 -cycle in $\mathscr{B}_{1 i}$ contains two edges that cannot occur in any other cycle of $\mathscr{B}_{1} \backslash \mathscr{B}_{1 i}$, which implies that such a 3-cycle is linearly independent with all the other 3 -cycles of $\mathscr{B}_{1} \backslash \mathscr{B}_{1 i}$. Therefore, $\mathscr{B}_{1}$ is linearly independent of 3 -cycles. Every cycle in $\mathscr{B}_{1}$ contains an edge of the form $\left(i, u_{j}\right)\left(i, u_{j+1}\right)$ for some $i$ and $j$, where $1 \leq i \leq n$ and $1 \leq j \leq 2 m-1$, that does not occur in any other cycle of $\mathscr{B}_{s}$. Thus, every cycle in $\mathscr{B}_{1}$ is linearly independent with all the other cycles in $\mathscr{B}_{s}$. Hence, $\mathscr{B}_{1} \cup \mathscr{B}_{s}$ is linearly independent.

For each $i, 1 \leq i \leq n, \mathscr{B}_{2 i}$ is a basis of the cycle subspace of $\mathscr{C}\left(P_{n}\left[L_{m}\right]\right)$ that corresponds to the $i$ th copy of the ladder $L_{m}$ which is obtained by pasting all the 4 -cycles in $\mathscr{B}_{2 i}$ successively at their common edges. Then $\mathscr{B}_{2 i}$ is linearly independent for each $i$. Furthermore, the cycles of $\mathscr{B}_{2 i}$ are edge-disjoint with all the cycles in $\mathscr{B}_{2} \backslash \mathscr{B}_{2 i}$ for every $i$. Therefore, $\mathscr{B}_{2}$ is linearly independent.

Now, every cycle in $\mathscr{B}_{2}$ contains an edge of the form $\left(i, u_{j}\right)\left(i, u_{2 m-j+1}\right), 1 \leq j \leq m-1$, which does not occur in any cycle of $\mathscr{B}_{1} \cup \mathscr{B}_{s}$. So, every cycle in $\mathscr{B}_{2}$ is linearly independent with all the cycles in $\mathscr{B}_{1} \cup \mathscr{B}_{s}$. Hence, $\mathscr{B}_{s} \cup \mathscr{B}_{1} \cup \mathscr{B}_{2}=\mathscr{B}_{(}\left(P_{n}\left[L_{m}\right]\right)$ is linearly independent. 
Moreover,

$$
\begin{aligned}
&\left|\mathscr{B}_{\left(P_{n}\left[L_{m}\right]\right) \mid}=\right| \mathscr{B}_{s}|+| \mathscr{B}_{1}|+| \mathscr{B}_{2} \mid \\
&=\sum_{i=1}^{n-1}\left|\mathscr{B}_{s i}\right|+\sum_{i=1}^{n-1}\left|\mathscr{B}_{1 i}\right|+\sum_{i=1}^{n}\left|\mathscr{B}_{2 i}\right| \\
&=(n-1)(2 m-1)^{2}+(n-1)(2(2 m-1))+n(m-1) \\
&=4 m^{2}(n-1)+m n-2 n+1=\operatorname{dim} \mathscr{C}\left(P_{n}\left[L_{m}\right]\right) .
\end{aligned}
$$

Since $\mathscr{B}\left(P_{n}\left[L_{m}\right]\right)$ is linearly independent and $\left|\mathscr{B}_{(}\left(P_{n}\left[L_{m}\right]\right)\right|=\operatorname{dim} \mathscr{C}\left(P_{n}\left[L_{m}\right]\right)$, then $\mathscr{B}\left(P_{n}\left[L_{m}\right]\right)$ is a basis of $\mathscr{C}\left(P_{n}\left[L_{m}\right]\right)$. It is an easy task to verify that $\mathscr{B}_{(}\left(P_{n}\left[L_{m}\right]\right)$ is a 4fold basis. Hence, $b\left(P_{n}\left[L_{m}\right]\right) \leq 4$ for all $n \geq 2$ and $m \geq 5$. On the other hand, to prove that $b\left(P_{n}\left[L_{m}\right]\right)=4$ for all $n \geq 2$ and $m \geq 7$, we need to exclude any possibility that the cycle space $\mathscr{C}\left(P_{n}\left[L_{m}\right]\right)$ has a 3 -fold basis for all $n \geq 2$ and $m \geq 7$. To this end, we suppose that $B$ is a 3 -fold basis of the cycle space $\mathscr{C}\left(P_{n}\left[L_{m}\right]\right)$ with $n \geq 2$ and $m \geq 7$. Then we consider the following three cases.

Case 1. Suppose that all the cycles in $B$ are 3 -cycles. Then, $|B| \leq 3(2 m-1) n+3 n(m-1)$ because any 3-cycle in $P_{n}\left[L_{m}\right]$ must contain exactly one edge from $E_{1} \cup E_{2}$ of fold at most 3. Thus, $4 m^{2}(n-1)+m n-2 n+1=|B| \leq 9 m n-6 n$, which implies that $4 m^{2}(n-1)+$ $1 \leq 8 m n-4 n$. Then, $m^{2} \leq 2 m(n /(n-1))-n /(n-1)-1 / 4(n-1)$. Since $n \geq 2$, we have $m^{2} \leq 4 m$, or $m \leq 4$, a contradiction.

Case 2. Suppose that all the cycles in $B$ have length greater than or equal to 4 . Then, by Lemma 1.2 , we have $4\left(4 m^{2}(n-1)+m n-2 n+1\right) \leq 3\left(4 m^{2}(n-1)+3 m n-2 n\right)$, which is equivalent to the inequality $16 m^{2}(n-1)+4 m n-8 n+4 \leq 12 m^{2}(n-1)+9 m n-6 n$. Rearranging this inequality implies that $4 m^{2}(n-1) \leq 5 m n+2 n-4$. Dividing by $4(n-1)$ gives $m^{2} \leq(5 m / 4)(n /(n-1))+(n-2) / 2(n-1)$. Since $n \geq 2$, we have $m^{2}<(5 m / 4)(n /(n$ $-1))<3 m$. Hence, $m<3$, a contradiction.

Case 3. Suppose that $B$ contains $s 3$-cycles and $t$ cycles of length greater than or equal to 4 . At most $3 s$ edges will be used to form the $s 3$-cycles, because the fold of every edge in $P_{n}\left[L_{m}\right]$ is at most 3. Then, $t \leq\left\lfloor\left(3\left(4 m^{2}(n-1)+3 m n-2 n\right)-3 s\right) / 4\right\rfloor$, where $\lfloor x\rfloor$ is the greatest integer less than or equal to $x$. Now, $4 m^{2}(n-1)+m n-2 n+1=|B|=$ $s+t \leq s+\left\lfloor\left(3\left(4 m^{2}(n-1)+3 m n-2 n\right)-3 s\right) / 4\right\rfloor \leq s+\left(3\left(4 m^{2}(n-1)+3 m n-2 n\right)-3 s\right) / 4$. Then, $16 m^{2}(n-1)+4 m n-8 n+4 \leq 4 s+12 m^{2}(n-1)+9 m n-6 n-3 s=s+12 m^{2}(n-$ $1)+9 m n-6 n$. But, as we have seen in Case $1, s \leq 9 m n-6 n$, so $16 m^{2}(n-1)+4 m n-$ $8 n+4 \leq 12 m^{2}(n-1)+18 m n-12 n$. This implies that $4 m^{2}(n-1) \leq 14 m n-4(n+1)$. Dividing by $4(n-1)$ implies that $m^{2} \leq(7 m / 2)(n /(n-1))-(n+1) /(n-1)<7 m$. Hence, $m<7$, a contradiction, because this inequality is not satisfied for all $m \geq 7$. This completes the proof.

We turn our attention to the graph $P_{n}\left[\mathrm{CL}_{m}\right]$ which is obtained from the graph of $P_{n}\left[L_{m}\right]$ by adding the following set of edges:

$$
E^{*}=\left\{\left(i, u_{m}\right)\left(i, u_{1}\right),\left(i, u_{m+1}\right)\left(i, u_{2 m}\right): 1 \leq i \leq n\right\}
$$


It is clear that $\left|E\left(P_{n}\left[\mathrm{CL}_{m}\right]\right)\right|=4 m^{2}(n-1)+3 m n,\left|V\left(P_{n}\left[\mathrm{CL}_{m}\right]\right)\right|=2 m$, and $\operatorname{dim} \mathscr{C}$ $\left(P_{n}\left[\mathrm{CL}_{m}\right]\right)=4 m^{2}(n-1)+m n+1$.

Theorem 2.2. For each $n \geq 2$ and $m \geq 5,3 \leq b\left(P_{n}\left[\mathrm{CL}_{m}\right]\right) \leq 4$. Moreover, $b\left(P_{n}\left[\mathrm{CL}_{m}\right]\right)=4$ for all $n \geq 2$ and $m \geq 7$.

Proof. Since $P_{n}\left[\mathrm{CL}_{m}\right]$ is nonplanar, MacLane's theorem implies that $b\left(P_{n}\left[\mathrm{CL}_{m}\right]\right) \geq 3$. To prove that $b\left(P_{n}\left[\mathrm{CL}_{m}\right]\right) \leq 4$, we have to prove that the set $\mathscr{B}\left(P_{n}\left[\mathrm{CL}_{m}\right]\right)=\mathscr{B}\left(P_{n}\left[L_{m}\right]\right) \cup \mathscr{B}^{*}$ is a 4 -fold basis for $\mathscr{C}\left(P_{n}\left[\mathrm{CL}_{m}\right]\right)$, where $\mathscr{B}\left(P_{n}\left[L_{m}\right]\right)$ is the 4 -fold basis of $\mathscr{C}\left(P_{n}\left[L_{m}\right]\right)$ which was constructed in Theorem 2.1 and $\mathscr{B}^{*}$ is defined as follows:

$$
\begin{aligned}
\mathscr{B}^{*}=\{ & \left(i+1, u_{1}\right)\left(i, u_{m}\right)\left(i, u_{1}\right)\left(i+1, u_{1}\right), \\
& \left.\left(i+1, u_{1}\right)\left(i, u_{m+1}\right)\left(i, u_{2 m}\right)\left(i+1, u_{1}\right) \mid 1 \leq i \leq n-1\right\} \cup\{a, b\},
\end{aligned}
$$

where $a$ and $b$ are two cycles given by

$$
\begin{aligned}
& a=\left(n-1, u_{2 m}\right)\left(n, u_{1}\right)\left(n, u_{m}\right)\left(n-1, u_{2 m}\right), \\
& b=\left(n-1, u_{2 m}\right)\left(n, u_{m+1}\right)\left(n, u_{2 m}\right)\left(n-1, u_{2 m}\right) .
\end{aligned}
$$

Every cycle in $\mathscr{B}^{*}$ contains an edge from $E^{*}$ that does not occur in any other cycle of $\mathscr{B}\left(P_{n}\left[\mathrm{CL}_{m}\right]\right)$. This means that every cycle in $\mathscr{B}^{*}$ is linearly independent with all the other cycles in $\mathscr{B}\left(P_{n}\left[\mathrm{CL}_{m}\right]\right)$. Thus, $\mathscr{B}\left(P_{n}\left[\mathrm{CL}_{m}\right]\right)$ is linearly independent. Moreover, $\left|\mathscr{B}\left(P_{n}\left[\mathrm{CL}_{m}\right]\right)\right|=\left|\mathscr{B}_{(}\left(P_{n}\left[L_{m}\right]\right)\right|+\left|\mathscr{B}^{*}\right|=\operatorname{dim} \mathscr{C}\left(P_{n}\left[\mathrm{CL}_{m}\right]\right)$. Hence, $\mathscr{B}\left(P_{n}\left[\mathrm{CL}_{m}\right]\right)$ is a basis of $\mathscr{C}\left(P_{n}\left[\mathrm{CL}_{m}\right]\right)$, and it is a simple matter to prove that $\mathscr{B}\left(P_{n}\left[\mathrm{CL}_{m}\right]\right)$ is 4 -fold.

On the other hand, to prove that $b\left(P_{n}\left[\mathrm{CL}_{m}\right]\right)=4$ for all $n \geq 2$ and $m \geq 7$, we have to prove that $\mathscr{C}\left(P_{n}\left[\mathrm{CL}_{m}\right]\right)$ cannot have any 3 -fold basis. To this end, we suppose that $B$ is a 3 -fold basis. Then, if we consider the three cases of Theorem 2.1 with similar arguments used there, taking into account that the number of 3-cycles in $B$ is at most $9 \mathrm{mn}$, we complete the proof.

We consider the graph of the Möbius ladder $\mathrm{ML}_{m}$ as a graph obtained from the circular ladder $\mathrm{CL}_{m}$ by deleting the edges $u_{m} u_{1}$ and $u_{2 m} u_{m+1}$ and replacing them by $u_{m+1} u_{1}$ and $u_{2 m} u_{m}$ respectively. Following these replacements, the graph of $P_{n}\left[\mathrm{ML}_{m}\right]$ is obtained from $P_{n}\left[\mathrm{CL}_{m}\right]$ by deleting all the edges $\left(i, u_{m}\right)\left(i, u_{1}\right)$ and $\left(i, u_{2 m}\right)\left(i, u_{m+1}\right)$ and replacing them by $\left(i, u_{m+1}\right)\left(i, u_{1}\right)$ and $\left(i, u_{2 m}\right)\left(i, u_{m}\right)$, respectively, for all $i$ with $1 \leq i \leq n$. Therefore, the proof of Theorem 2.2 works word by word for the following theorem after making the replacements of the corresponding edges in the cycles of the set $\mathscr{B}\left(P_{n}\left[\mathrm{CL}_{m}\right]\right)$ in Theorem 2.2.

Theorem 2.3. For each $n \geq 2$ and $m \geq 5,3 \leq b\left(P_{n}\left[\mathrm{ML}_{m}\right]\right) \leq 4$. Moreover, $b\left(P_{n}\left[\mathrm{ML}_{m}\right]\right)=$ 4 for all $n \geq 2$ and $m \geq 7$.

Now, for the graph $C_{n}\left[L_{m}\right]$, we have $\left|V\left(C_{n}\left[L_{m}\right]\right)\right|=2 m n,\left|E\left(C_{n}\left[L_{m}\right]\right)\right|=4 m^{2} n+3 m n$ $-2 n$, and $\operatorname{dim} \mathscr{C}\left(C_{n}\left[L_{m}\right]\right)=4 m^{2} n+m n-2 n+1$. We consider the graph $C_{n}\left[L_{m}\right]$ as a graph obtained from the graph $P_{n}\left[L_{m}\right]$, which is defined in Theorem 2.1, by adding a new copy of $K_{2 m, 2 m}$ from the set of vertices $\left\{\left(n, u_{j}\right): 1 \leq j \leq 2 m\right\}$ and the set of vertices $\left\{\left(1, u_{j}\right): 1 \leq j \leq 2 m\right\}$. So, we have $C_{n}\left[L_{m}\right]=P_{n}\left[L_{m}\right] \cup\left\{\left(n, u_{j}\right)\left(1, u_{k}\right): 1 \leq j, k \leq 2 m\right\}$. 
Theorem 2.4. For each $n \geq 3$ and $m \geq 5,3 \leq b\left(C_{n}\left[L_{m}\right]\right) \leq 4$. Moreover, $b\left(C_{n}\left[L_{m}\right]\right)=4$ for all $n \geq 4$ and $m \geq 7$.

Proof. The graph $C_{n}\left[L_{m}\right]$ is nonplanar for all $n \geq 3$ and $m \geq 5$, then by MacLane's theorem, we have $b\left(C_{n}\left[L_{m}\right]\right) \geq 3$. To prove that $b\left(C_{n}\left[L_{m}\right]\right) \leq 4$, we exhibit a 4 -fold basis for $\mathscr{C}\left(C_{n}\left[L_{m}\right]\right)$. Define

$$
\mathscr{B}\left(C_{n}\left[L_{m}\right]\right)=\mathscr{B}_{(}\left(P_{n}\left[L_{m}\right]\right) \cup \mathscr{B}_{s n} \cup \mathscr{B}_{1 n} \cup\{c\}
$$

where

$$
\begin{gathered}
\mathscr{B}_{s n}=\left\{\left(n, u_{j}\right)\left(1, u_{k}\right)\left(n, u_{j+1}\right)\left(1, u_{k+1}\right)\left(n, u_{j}\right): 1 \leq j, k \leq 2 m-1\right\}, \\
\mathscr{B}_{1 n}=\left\{\left(n, u_{1}\right)\left(1, u_{j}\right)\left(1, u_{j+1}\right)\left(n, u_{1}\right),\left(1, u_{2 m}\right)\left(n, u_{j}\right)\left(n, u_{j+1}\right)\left(1, u_{2 m}\right): 1 \leq j \leq 2 m-1\right\},
\end{gathered}
$$

and the cycle $c$ is given by

$$
c=\left(1, u_{1}\right)\left(2, u_{1}\right) \cdots\left(n-1, u_{1}\right)\left(n, u_{1}\right)\left(1, u_{1}\right)
$$

The set $\mathscr{B}\left(P_{n}\left[L_{m}\right]\right)$ is linearly independent, since it is the required 4 -fold basis of $\mathscr{C}\left(P_{n}\left[L_{m}\right]\right)$ which was constructed in Theorem 2.1 . The sets $\mathscr{S}_{s n}$ and $\mathscr{B}_{1 n}$ are linearly independent for the same reasons stated in the proof of Theorem 2.1 where we proved that the sets $\mathscr{S}_{s i}$ and $\mathscr{S}_{1 i}$ are linearly independent. Also, using similar arguments to those used in Theorem 2.1, we conclude that $\mathscr{S}_{(}\left(P_{n}\left[L_{m}\right]\right) \cup \mathscr{B}_{s n} \cup \mathscr{B}_{1 n}$ is linearly independent. Moreover, every edge in the $n$-cycle $c$ belongs to one of the $n$ copies of the graph $K_{2 m, 2 m}$, and since all these copies are edge-disjoint, then $c$ must be linearly independent with all the other cycles of $\mathscr{B}\left(C_{n}\left[L_{m}\right]\right)$. Hence, $\mathscr{B}\left(C_{n}\left[L_{m}\right]\right)$ is linearly independent. Furthermore, we have

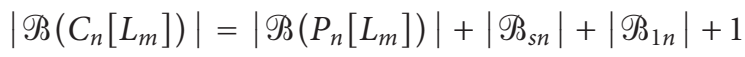

$$
\begin{aligned}
& =4 m^{2}(n-1)+m n+1+(2 m-1)^{2}+(4 m-2)+1 \\
& =4 m^{2} n+m n-2 n+1=\operatorname{dim} \mathscr{b}\left(C_{n}\left[L_{m}\right]\right) \text {. }
\end{aligned}
$$

Therefore, $\mathscr{B}\left(C_{n}\left[L_{m}\right]\right)$ is a basis, and verifying that it is a 4 -fold basis is a simple matter.

On the other hand, to prove that $b\left(C_{n}\left[L_{m}\right]\right)=4$ for all $n \geq 4$ and $m \geq 7$, we prove that $\mathscr{C}\left(C_{n}\left[L_{m}\right]\right)$ cannot have any 3 -fold basis. To this end, we suppose that $B$ is a 3 -fold basis of $\mathscr{C}\left(C_{n}\left[L_{m}\right]\right)$, then using similar arguments to those used in the three cases of Theorem 2.1, we get the required contradiction.

For the graph $C_{n}\left[\mathrm{CL}_{m}\right],\left|V\left(C_{n}\left[\mathrm{CL}_{m}\right]\right)\right|=2 m n,\left|E\left(C_{n}\left[\mathrm{CL}_{m}\right]\right)\right|=4 m^{2} n+3 m n$, and $\operatorname{dim} \mathscr{C}\left(C_{n}\left[\mathrm{CL}_{m}\right]\right)=4 m^{2} n+m n+1$. We consider the graph of $C_{n}\left[\mathrm{CL}_{m}\right]$ as a graph obtained from the graph $P_{n}\left[\mathrm{CL}_{m}\right]$ by adding the set of edges $E^{*}$ which we have defined after Theorem 2.1. 
Theorem 2.5. For each $n \geq 4$ and $m \geq 5, b\left(C_{n}\left[\mathrm{CL}_{m}\right]\right)=4$.

Proof. To prove that $b\left(C_{n}\left[\mathrm{CL}_{m}\right]\right) \leq 4$ for all $n \geq 4$ and $m \geq 5$, we look for a 4 -fold basis for the cycle space $\mathscr{C}\left(C_{n}\left[\mathrm{CL}_{m}\right]\right)$. Define the set $\mathscr{B}\left(C_{n}\left[\mathrm{CL}_{m}\right]\right)=\mathscr{B}\left(C_{n}\left[L_{m}\right]\right) \cup \mathscr{B}^{*}$, where $\mathscr{B}\left(C_{n}\left[L_{m}\right]\right)$ is the basis of $\mathscr{C}\left(C_{n}\left[L_{m}\right]\right)$ which was constructed in Theorem 2.4 and $\mathscr{B}^{*}$ is the set of cycles that we have defined previously in Theorem 2.2. Since we have seen that each of these sets is linearly independent and every cycle in $\mathscr{B}^{*}$ contains an edge that does not occur in any other cycle in $\mathscr{B}\left(C_{n}\left[L_{m}\right]\right)$, we conclude that $\mathscr{B}\left(C_{n}\left[\mathrm{CL}_{m}\right]\right)$ is linearly independent. Moreover, we have

$$
\begin{aligned}
\left|\mathscr{B}\left(C_{n}\left[\mathrm{CL}_{m}\right]\right)\right| & =\left|\mathscr{B}\left(C_{n}\left[L_{m}\right]\right)\right|+\left|\mathscr{B}^{*}\right| \\
& =\left(4 m^{2} n+m n-2 n+1\right)+2 n \\
& =\operatorname{dim} \mathscr{C}\left(C_{n}\left[\mathrm{CL}_{m}\right]\right) .
\end{aligned}
$$

Hence, $\mathscr{B}\left(C_{n}\left[\mathrm{CL}_{m}\right]\right)$ is a basis of $\mathscr{C}\left(C_{n}\left[\mathrm{CL}_{m}\right]\right)$ and it can be proved easily that it is a 4 -fold basis.

On the other hand, to prove that $b\left(C_{n}\left[\mathrm{CL}_{m}\right]\right) \geq 4$ for all $n \geq 4$ and $m \geq 5$, we use contradiction to eliminate any possibility that $\mathscr{C}\left(C_{n}\left[\mathrm{CL}_{m}\right]\right)$ has a 3 -fold basis, in fact, using similar arguments to those used in the cases of Theorem 2.1.

Finally, we consider $C_{n}\left[\mathrm{ML}_{m}\right]$ as a graph obtained from $C_{n}\left[\mathrm{CL}_{m}\right]$ by deleting the following set of edges:

$$
B^{* *}=\left\{\left(i, u_{m}\right)\left(i, u_{1}\right),\left(i, u_{2 m}\right)\left(i, u_{m+1}\right): 1 \leq i \leq n\right\}
$$

and replacing it with the following set of edges:

$$
B^{* * *}=\left\{\left(i, u_{m+1}\right)\left(i, u_{1}\right),\left(i, u_{2 m}\right)\left(i, u_{m}\right): 1 \leq i \leq n\right\}
$$

Following these replacements of edges, we can repeat the proof of Theorem 2.5 to prove the following theorem.

Theorem 2.6. For each $n \geq 4$ and $m \geq 5, b\left(C_{n}\left[\mathrm{ML}_{m}\right]\right)=4$.

\section{References}

[1] A. A. Ali, The basis numbers of the direct products of paths and cycles, Ars Combin. 27 (1989), 155-163.

[2] A. A. Ali and G. T. Marougi, The basis number of the lexicographic product of graphs, Ars Combin. 36 (1993), 271-282.

[3] S. Y. Alsardary and J. Wojciechowski, The basis number of the powers of the complete graph, Discrete Math. 188 (1998), no. 1-3, 13-25.

[4] M. Y. Alzoubi and M. M. M. Jaradat, The basis number of the composition of theta graphs with stars and wheels, Acta Math. Hungar. 103 (2004), no. 3, 255-263.

[5] J. A. Banks and E. F. Schmeichel, The basis number of the n-cube, J. Combin. Theory Ser. B 33 (1982), no. 2, 95-100.

[6] J. A. Bondy and U. S. R. Murty, Graph Theory with Applications, American Elsevier, New York, 1976. 


\section{Basis number of composition of graphs}

[7] M. Q. Hailat and M. Y. Alzoubi, The basis number of the composition of graphs, İstanbul Üniv. Fen Fak. Mat. Derg. 53 (1994), 43-60 (1996).

[8] F. Harary, Graph Theory, Addison-Wesley, Massachusetts, 1969.

[9] M. M. M. Jaradat, On the basis number of the direct product of graphs, Australas. J. Combin. 27 (2003), 293-306.

[10] M. M. M. Jaradat and M. Y. Alzoubi, On the basis number of the semi-strong product of bipartite graphs with cycles, Kyungpook Math. J. 45 (2005), 45-53.

[11] S. MacLane, A combinatorial condition for planar graphs, Fund. Math. 28 (1937), 22-32.

[12] E. F. Schmeichel, The basis number of a graph, J. Combin. Theory Ser. B 30 (1981), no. 2, 123129.

Maref Y. Alzoubi: Department of Mathematics, Faculty of Science, Yarmouk University, Irbid, Jordan

E-mail address: maref@yu.edu.jo

M. M. M. Jaradat: Department of Mathematics, Faculty of Science, Yarmouk University, Irbid, Jordan

E-mail address: mmjst4@yu.edu.jo 


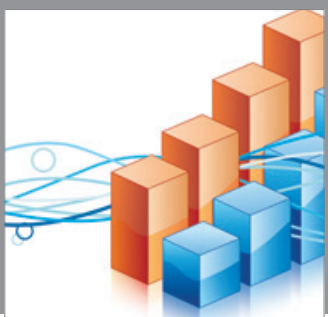

Advances in

Operations Research

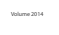



\section{The Scientific} World Journal
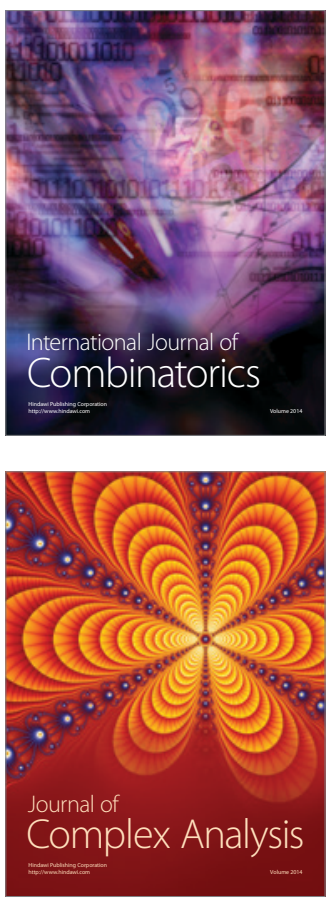

International Journal of

Mathematics and

Mathematical

Sciences
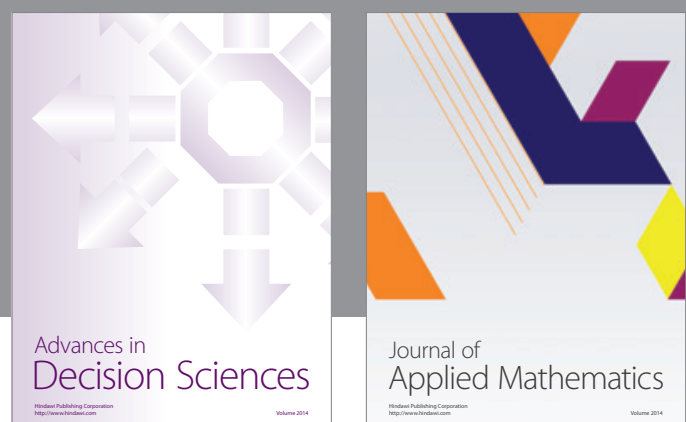

Journal of

Applied Mathematics
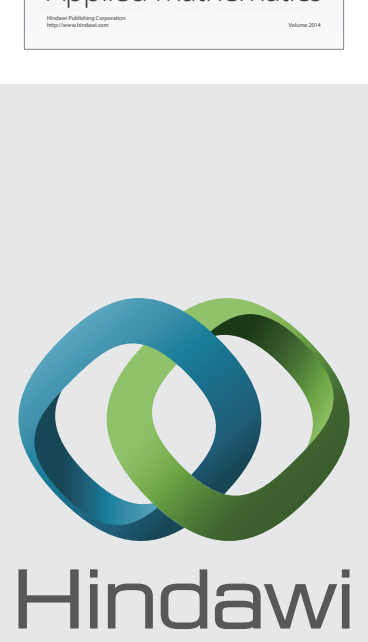

Submit your manuscripts at http://www.hindawi.com
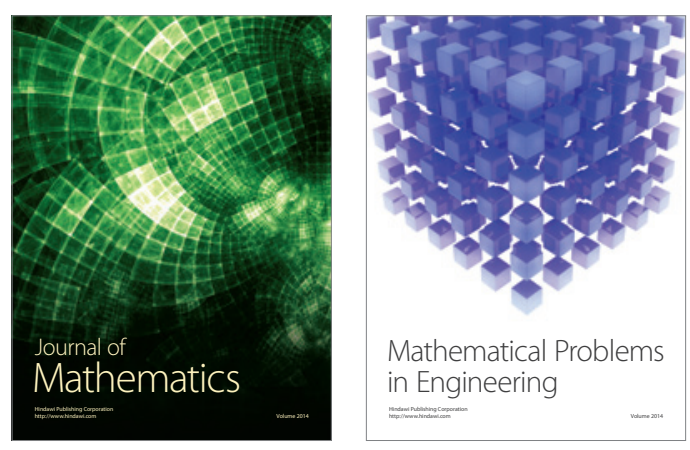

Mathematical Problems in Engineering
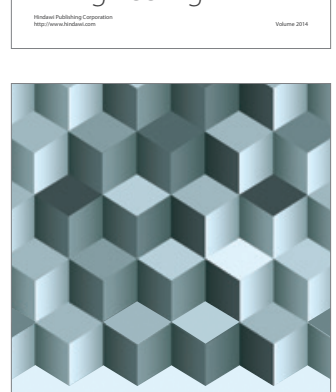

Journal of

Function Spaces
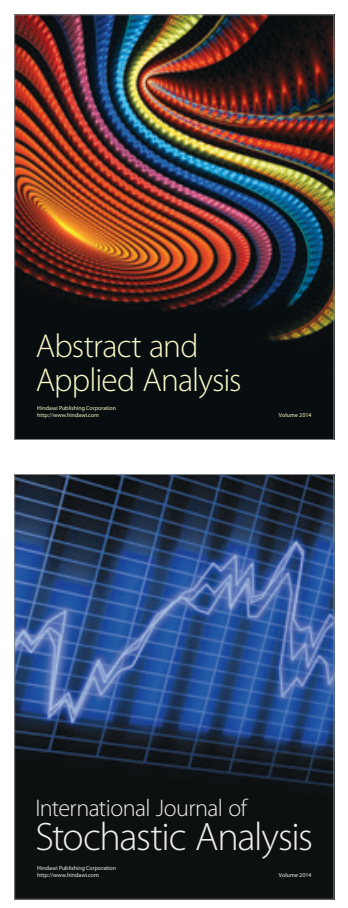

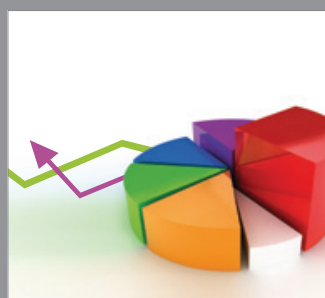

ournal of

Probability and Statistics

Promensencen
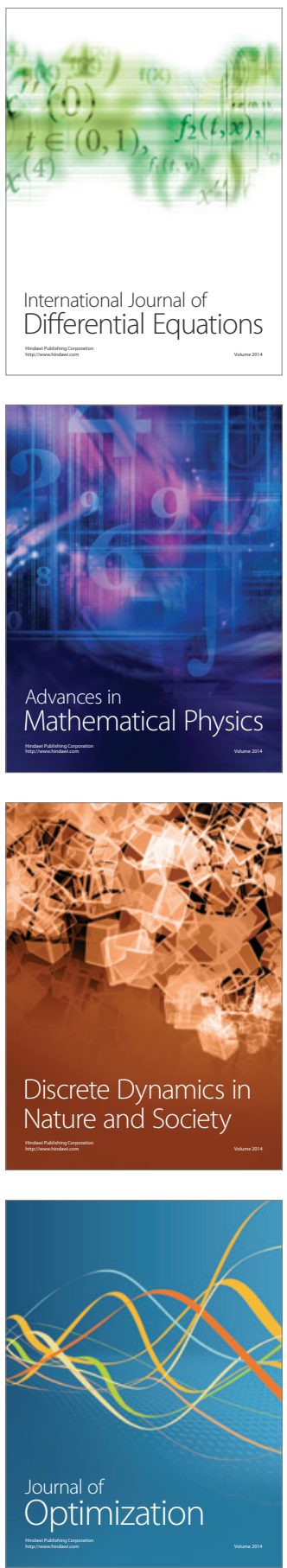